\title{
What predicts falls in Parkinson disease? \\ Observations from the Parkinson's Foundation registry
}

Sotirios A. Parashos, MD, Bastiaan R. Bloem, MD, Nina M. Browner, MD, Nir Giladi, MD, Tanya Gurevich, MD, Jeffrey M. Hausdorff, PhD, Ying He, PhD, Kelly E. Lyons, PhD, Zoltan Mari, MD, John C. Morgan, MD, Bart Post, MD, Peter N. Schmidt, PhD, Catherine L. Wielinski, MPH, and Samuel S. Wu, PhD, On behalf of The National Parkinson Foundation Quality Improvement Initiative Investigators

Neurology: Clinical Practice June 2018 vol. 8 no. 3 214-222 doi:10.1212/CPJ.0000000000000461
Correspondence

Dr. Parashos

sotirios.parashos@parknicollet.com

\begin{abstract}
\section{Background}

We undertook this study to identify patients with Parkinson disease (PD) with no or rare falls who may progress to frequent falling by their next annual follow-up visit.
\end{abstract}

\section{Methods}

We analyzed data in the National Parkinson Foundation Quality Improvement Initiative database to identify factors predicting which patients with PD with no or rare falls at the baseline visit will report at least monthly falls at the annual follow-up visit. Multivariable models were constructed using logistic regression. Variables were introduced in 4 blocks: in the 1st block, variables present at or before the baseline visit were entered; in the 2nd, baseline visit assessments; in the 3rd, interventions implemented during baseline visit; and, in the 4th block, changes in comorbidities, living situation, and treatment between visits.

\section{Results}

Of 3,795 eligible participants, 3,276 (86.3\%) reported no or rare falls at baseline visit, and of them, $382(11.7 \%)$ reported at least monthly falls at follow-up visit. Predictors included female sex, $<90 \%$ diagnostic certainty, motor fluctuations, levodopa treatment, antidepressant treatment, prior deep brain stimulation (DBS), worse quality of life, Hoehn \& Yahr stage 2 or 3 , worse semantic fluency, and, between visits, addition of amantadine, referral to occupational therapy, social services, or DBS, new diagnoses of cancer or osteoarthritis, and increased emergency visits.

\section{Conclusions}

This large-scale analysis identified several predictors of progression to falling in PD. Such identifiers may help target patient subgroups for falls prevention intervention. Some factors are modifiable, offering opportunities for developing such interventions.

Struthers Parkinson's Center (SAP, CLW), HealthPartners, Golden Valley, MN; Parkinson Center Nijmegen (BRB, BP), Donders Institute for Brain, Cognition and Behavior, Radboud University Medical Center, Department of Neurology, the Netherlands; Department of Neurology (NMB), University of North Carolina at Chapel Hill; Neurological Institute (NG, TG, JMH), Tel Aviv Sourasky Medical Center, Sackler School of Medicine, and Sagol School of Neuroscience, Tel-Aviv University, Israel; Department of Mathematics (YH), Clarkson University, Potsdam, NY; University of Kansas Medical Center Parkinson's Disease Center (KEL), Kansas City; Department of Neurology (ZM), Johns Hopkins University, Baltimore, MD, currently at Cleveland Clinic Lou Ruvo Center for Brain Health, Las Vegas, NV; Movement Disorders Program (JCM), NPF Center of Excellence, Department of Neurology, Medical College of Georgia, Augusta University; Parkinson's Foundation (PNS), Miami, FL; and Department of Biostatistics (SSW), University of Florida, Gainesville.

Funding information and disclosures are provided at the end of the article. Full disclosure form information provided by the authors is available with the full text of this article at Neurology.org/cp.

Coinvestigators are listed at links.Iww.com/CPJ/A29. 
In this study, we identified history of DBS prior to baseline visit, and also DBS implemented between visits as

\section{a significant risk factor of conversion \\ to frequent faller status.}

Falls considerably augment disease burden in Parkinson disease (PD). Approximately $50 \%$ of patients with PD who fall require medical care. ${ }^{1}$ Falls increase PD morbidity and mortality, ${ }^{2-4}$ caregiver burden, ${ }^{5}$ and utilization and cost of health care, ${ }^{6}$ and reduce quality of life. ${ }^{7}$

Developing and optimizing therapeutic strategies to prevent falls requires identifying patients with PD at risk for falling. Prior history of falls is the most important such risk factor in $\mathrm{PD}$, yet roughly $25 \%$ of patients with PD who fell in prospective studies were first-time fallers. ${ }^{8}$ Other risk factors include age, disease duration, disease stage, absence of rest tremor, severity of motor impairment, postural instability and gait disorder, cognitive dysfunction, comorbidities, antidepressants, and deep brain stimulation (DBS) ${ }^{1,9-11}$ Recent studies have identified useful clinical variables and tests in predicting falls among nonfallers in PD, such as intensity of dopaminergic treatment, frontal dysfunction, and disease severity ${ }^{12}$; gait characteristics ${ }^{13}$; the miniBESTest ${ }^{14}$; and higher dual task cost. ${ }^{15}$ As part of the Quality Improvement Initiative (QII) of the National Parkinson Foundation (NPF), data were collected annually in a large sample of PD outpatients from 19 NPF Centers of Excellence. ${ }^{16}$ We previously presented a crosssectional analysis of risk factors for falls in a subset of the baseline data from this registry. ${ }^{11}$

In this article, we analyze longitudinal data from the NPF-QII registry to address the following question: What characteristics identify those most likely to become frequent fallers among nonfalling or rarely falling patients with PD?

\section{Methods}

\section{Standard protocol approvals, registrations, and patient consents}

Data for this analysis were obtained from the NPF-QII registry (ClinicalTrials.gov protocol number NCT01629043). We utilized available data as of February 2015. Data collection was approved by the institutional review boards of participating sites, and all participants signed written informed consent prior to participation. In brief, patients with PD followed at 19 clinics designated NPF Centers of Excellence were recruited in a longitudinal observational registry. Every year a battery of outcomes is collected for each participant in a standardized fashion. Although this visit happens on an annual basis, the interval between visits is variable. The details of instrument selection and methods of data collection have been previously published. ${ }^{16}$

\section{Assessments}

Data extracted for this analysis from the NPF-QII registry included the following:

1. Demographics: age at baseline visit, sex, race, living arrangements, presence of regular care partner, study site.

2. Medical history: disease duration at baseline visit; investigator's confidence in the diagnosis; presence of rest tremor; presence of motor fluctuations; history of falls over the preceding 3 months; comorbidities; treatment with levodopa, dopamine agonists, monoamine oxidase type B inhibitors, catechol-O-methyl transferase inhibitors, amantadine, anticholinergics, antidepressants, antipsychotics, and cognitive enhancers; history of DBS for PD; hospitalizations in the preceding year; physical, occupational, and speech therapy, exercise, social or mental health services.

3. Objective and self-reported measures of impairment and quality of life at baseline visit: Parkinson's Disease Questionnaire (PDQ-39) ${ }^{17}$; Hoehn \& Yahr (H\&Y) stage $^{18}$; Timed Up-and-Go (TUG) $)^{19}$; modified Multidimensional Caregiver Strain Index $(\mathrm{MCSI})^{20}$; immediate and delayed recall of 5 words (from version 1 of the English language Montreal Cognitive Assessment ${ }^{21}$; semantic fluency (number of animals recited in 1 minute).

4. Changes in treatment at baseline visit: new addition or discontinuation of any of the above classes of medications, new referrals for any of the above mentioned treatment modalities.

5. Changes in medical history between visits: changes in comorbidities, hospitalizations, or living situation between baseline and follow-up visits.

6. Time interval between visits: visits are annual, but the interval between visits is variable, and therefore the interval is included as an independent variable.

\section{Statistical design, primary outcome, sample selection and analysis}

In the NPF QII database, the "falls" variable is stratified as $0=$ none, $1=$ rare, $2=$ monthly, $3=$ weekly, $4=$ daily. The purpose of this analysis was to identify risk factors predicting that a participant with no or rare falls at baseline visit will report at least monthly falls at the annual follow-up visit. Therefore we included in the analysis all registry enrollees who reported no or rare falls at baseline. The primary outcome was "Frequent faller (yes/no)" at the time of followup. A frequent faller was defined as a participant reporting at least monthly falls in the preceding 3 months.

In evaluating candidate predictor variables, we proceeded in a stepwise fashion, whereby blocks of variables were hierarchically added to the building of the final model. The rationale for this process was that risk factors may have different 
predictive implications and different potential for modification. In addition, this process helped streamline the progression of the analysis in a logical manner. Thus, the first block analyzed variables that were already present and unalterable at the time of baseline visit (such as disease duration, or age, or comorbidities at baseline); the second block started with the significant variables from the first block with the addition of the candidate predictor variables from assessments performed at baseline. In block 3, the model was further augmented by adding the treatments ordered at baseline visit, with the final, fourth block exploring the contribution of comorbidities and hospitalizations that occurred between visits. Candidate explanatory variables are listed by block in table e-1 (links.lww.com/CPJ/A28). At each level, univariate analyses were first performed using $\chi^{2}$ or $t$ test, followed by the building of multivariable models, using backward selection with a significance level of 0.1 to keep a risk factor in subsequent models. In a separate analysis we looked for predictors among the assessments performed during baseline visit, without accounting for any of the other variables. The purpose of this analysis was to assess the predictive value of the instruments regardless of demographic or other disease and comorbidity variables.

The above statistical analyses were performed using SAS version 9.3. All individual tests were 2 -sided and $p$ values less than 0.05 were deemed statistically significant.

\section{Data availability}

Specified anonymized data will be shared by request from any qualified investigator for the purpose of confirming or replicating our findings.

\section{Results}

\section{Study sample, demographics, disease characteristics, and objective assessments}

Because the collection of information on falls was implemented as an amendment to the original protocol, baseline falls data were available on 6,382 out of a total of 7,846 enrolled patients, and among them 3,815 had completed the annual follow-up visit. Due to missing data on the falls question, 20 patients were excluded from further study. Of the remaining 3,795 participants, 3,276 (86.3\%) (aged 65.6 \pm 9.8 at baseline, $36.5 \%$ female) reported no or rare falls in the preceding 3 months and were included in the analysis (figure). For predictive statistics, only participants with complete datasets were utilized, which provided a sample size of 2,760 (or $84.3 \%$ of the study sample).

\section{Relationship between demographics, disease characteristics, objective assessments, and main outcome measure}

Out of 3,276 nonfallers/rare fallers at baseline, 382 (11.7\%) became frequent fallers by the annual follow-up visit, which leads to an annual rate of conversion of $8.9 \%$ (accounting
Semantic fluency was more strongly associated with increased risk for becoming a frequent faller than 5word retention and recall.

for an average follow-up interval of $481.1 \pm 199.8$ days). Table 1 shows the demographics and disease characteristics of the study sample, categorized by the primary outcome, i.e., none or rare falls vs monthly or more frequent falls at follow-up. In a similar fashion, table e-2 (links.lww.com/ $\mathrm{CPJ} / \mathrm{A} 28$ ) includes additional demographic detail, table e-3 summarizes comorbidities, table e- 4 treatments in place at baseline, and table e-5 changes in treatments and new therapies or services ordered or implemented during the 3 months preceding the annual follow-up visit for the same sample.

\section{Univariate analyses}

Significant differences in baseline characteristics were found between the 2 fall status groups, and are listed in table 1 and tables e-2 through e-5 (links.lww.com/CPJ/A28). In addition, there was a difference in the main outcome across centers $(p<0.001)$. All identified differences remain statistically significant at the 0.05 level after Bonferroni adjustment is applied to the 15 tests.

\section{Multivariate models}

Table 2 summarizes the final multivariate model that predicts conversion to frequent faller status between visits. The coefficient of determination $\left(R^{2}\right)$ of the final model was $21.10 \%$. Tables e-6, e-7, and e-8 (links.lww.com/CPJ/ A28) show the progression of model building with each successive block. To assess how the model can predict other datasets, we performed 1,000 cross-validations with $10 \%$ samples left out each time. The overall accuracy has a median of $69 \%$ and interquartile range of $67 \%-71 \%$, while the median sensitivity and specificity were $70 \%$ and $69 \%$, respectively. Table 3 shows separately the predictive value of the questionnaires and objective measures obtained at baseline, without accounting for any other variables. The $R^{2}$ for the model of the predictive value of the assessments was $13.63 \%$.

\section{Discussion}

This study is one of 2 large-scale observational analyse ${ }^{22}$ of progression of falls in $\mathrm{PD}$, and expands the conclusions of previous cross-sectional studies of falling in PD. Among the most striking findings are the relative importance of a measure of frontal cognitive dysfunction over a measure of memory, the predictive value of motor fluctuations, the 
importance of emerging comorbidities, and the complex nature of DBS as a factor in the genesis of falls in PD. Frequent falls emerged in a setting of increasing morbidity manifested by increasing needs for hospital and allied health services, and social support. Not surprisingly, increasing disease severity and decreasing health-related quality of life strongly correlated with progression to frequent falls. Despite the extent of the data collected and analyzed, the coefficient of determination for the final model was low (approximating 21\%).

The rate of conversion to frequent faller in our study (8.9\%) agrees with previously reported annual rates of conversion of nonfaller to faller of approximately $10 \%,{ }^{22}$ and frequent faller of $19 \%,{ }^{23}$ despite different methodologies.

Female sex and older age have been variably associated with increased fall risk in PD. ${ }^{1,11}$ One conceivable explanation for such variability is the observation ${ }^{22}$ (confirmed in our cohort) that there is an interaction between age and sex. Further investigation of this interaction indicated that as age increases, the effect of sex abates. ${ }^{22}$

The significance of "certainty of diagnosis" in our analysis is of questionable value. Atypical parkinsonian syndromes are associated with a higher risk of falling, ${ }^{1}$ and it is possible that the presence of postural instability (a determinant of the primary outcome) may have influenced the certainty of PD diagnosis. A notable negative finding was the absence of rest tremor as a protective risk factor against falling. This may be more in line with recent indications that a postural instability/gait difficulty predominant phenotype is a risk factor for more rapid PD progression, rather than that rest tremor is a protective factor. ${ }^{24}$

Motor fluctuations are rarely reported as a prognostic factor of falls. This may reflect the fact that motor fluctuations have not been considered in previous analyses, or that motor fluctuations are a surrogate for disease severity. Since it would be highly unusual for a patient to exhibit disease severity sufficient to lead to frequent falls, yet insufficient to warrant treatment with levodopa, the finding of levodopa treatment predicting increasing falls is not surprising.

We have previously reported an association between antidepressants and fall risk, ${ }^{11}$ and the same association has been observed in an analysis of a large clinical trial cohort. ${ }^{22}$ Antidepressants are a known falls risk factor in older adults. ${ }^{25,26}$ It is conceivable that antidepressants constitute a surrogate variable for depression, a risk factor for falling in the general population, ${ }^{27}$ also associated with axial symptoms in PD. ${ }^{28}$ Nevertheless, the finding merits attention and consideration should be given to nonmedicinal approaches to the treatment of depression in patients with PD at risk of falling.

Figure Study sample selection

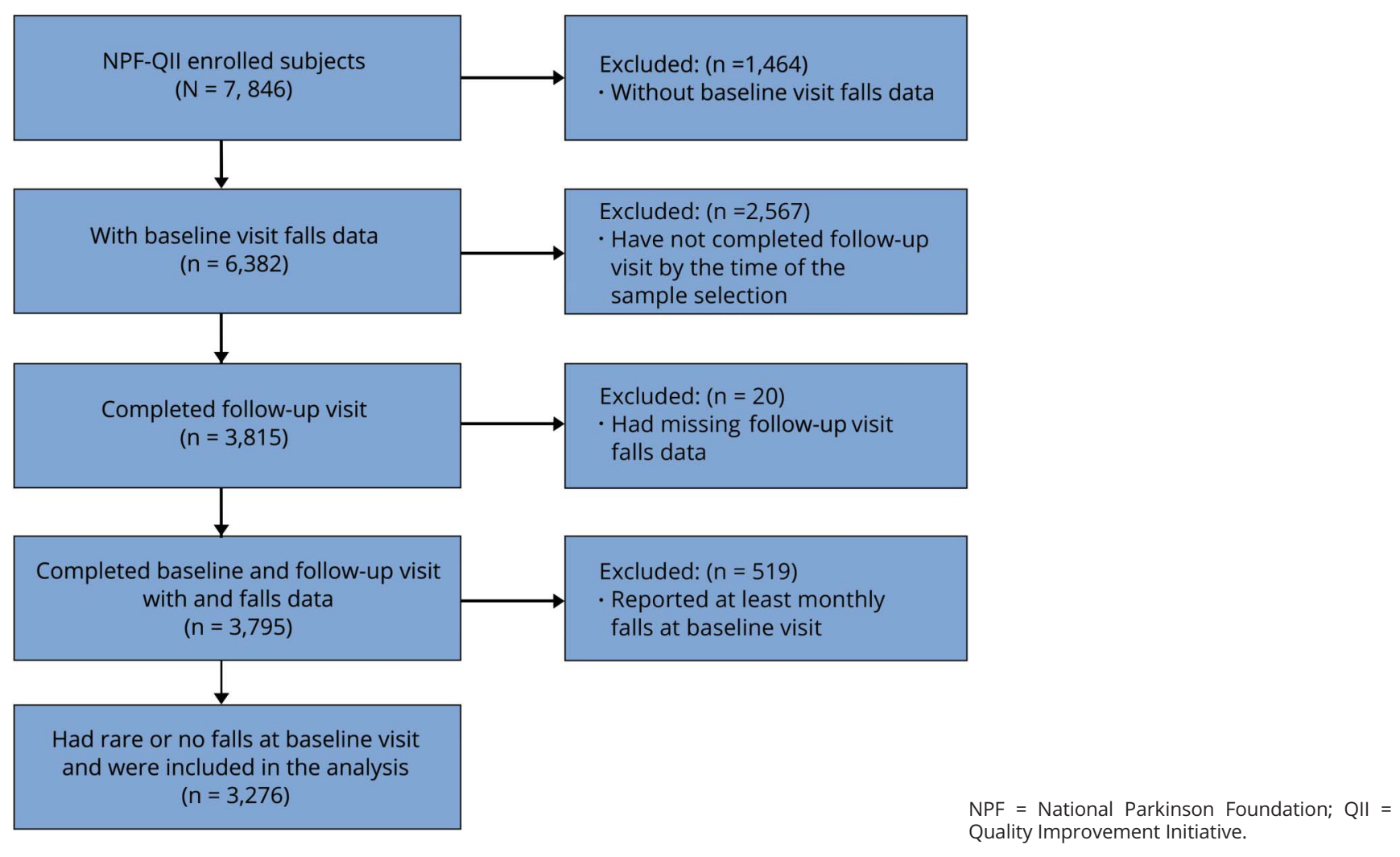


Table 1 Demographic and clinical characteristics at baseline visit

\begin{tabular}{|c|c|c|c|c|}
\hline \multirow[b]{2}{*}{ Variables } & \multicolumn{2}{|c|}{ Frequent faller at follow-up } & \multirow[b]{2}{*}{$p$ Value } & \multirow[b]{2}{*}{ Missing rate } \\
\hline & No $(n=2,894)$ & Yes $(n=382)$ & & \\
\hline \multicolumn{5}{|l|}{ Sex } \\
\hline Male & $1839(63.6)$ & $239(62.6)$ & 0.702 & $1(0.03)$ \\
\hline Female & $1054(36.4)$ & $143(37.4)$ & & \\
\hline Age at diagnosis, y & $65.4 \pm 9.8$ & $67.2 \pm 9.6$ & 0.001 & 0 \\
\hline Disease duration, y & $7.5 \pm 5.5$ & $10.1 \pm 6.1$ & $<0.0001$ & $1(0.03)$ \\
\hline Time between visits, $d$ & $479.7 \pm 200.7$ & $491.7 \pm 192.9$ & 0.268 & 0 \\
\hline \multicolumn{5}{|l|}{ Certainty of diagnosis } \\
\hline$<90 \%$ & $321(11.2)$ & $53(14.0)$ & 0.108 & $26(0.79)$ \\
\hline$\geq 90 \%$ & $2550(88.8)$ & $326(86.0)$ & & \\
\hline \multicolumn{5}{|l|}{ Rest tremor } \\
\hline No & $806(28.0)$ & $120(31.5)$ & 0.155 & $16(0.49)$ \\
\hline Yes & $2073(72.0)$ & $261(68.5)$ & & \\
\hline \multicolumn{5}{|l|}{ Motor fluctuations } \\
\hline No & $1730(60.0)$ & $152(40.2)$ & $<0.0001$ & $16(0.49)$ \\
\hline Yes & $1152(40.0)$ & $226(59.8)$ & & \\
\hline \multicolumn{5}{|l|}{ H\&Y stage } \\
\hline 1 & $469(16.2)$ & $16(4.2)$ & $<0.0001$ & $257(7.84)$ \\
\hline 2 & $1595(55.1)$ & $166(43.5)$ & & \\
\hline 3 & $522(18.0)$ & $143(37.4)$ & & \\
\hline 4-5 & $83(2.9)$ & $25(6.5)$ & & \\
\hline Not assessed & $225(7.8)$ & $32(8.4)$ & & \\
\hline No. of comorbidities & $1.3 \pm 1.2$ & $1.5 \pm 1.3$ & 0.003 & $2(0.06)$ \\
\hline PDQ-39 summary score (\%) & $20.6 \pm 13.8$ & $30.2 \pm 14.4$ & $<0.0001$ & $18(0.55)$ \\
\hline MCSI (\%) & $14.1 \pm 13.6$ & $23.0 \pm 15.7$ & $<0.0001$ & $1414(43.16)$ \\
\hline TUG & $11.9 \pm 6.0$ & $14.7 \pm 7.7$ & $<0.0001$ & $139(4.24)$ \\
\hline Immediate 5-word recall & $4.5 \pm 0.8$ & $4.2 \pm 1.0$ & $<0.0001$ & $32(0.98)$ \\
\hline Semantic fluency & $19.5 \pm 6.4$ & $16.3 \pm 5.8$ & $<0.0001$ & $48(1.47)$ \\
\hline Delayed (5-min) 5-word recall & $3.3 \pm 1.4$ & $3.0 \pm 1.3$ & $<0.001$ & $46(1.4)$ \\
\hline
\end{tabular}

Abbreviations: H\&Y = Hoehn \& Yahr; PDQ-39 = 39-item Parkinson's Disease Questionnaire; MSCI = Modified Caregiver Strain Index; TUG = Timed-Up-and-Go. Values are $\mathrm{n}(\%)$ or mean \pm SD.

The association between anticholinergic burden and falling has been a subject of recent studies, ${ }^{29}$ and a clinical trial has demonstrated a reduction of falls among patients with PD who were treated with a centrally acting anticholinesterase. ${ }^{30}$ In our analysis, anticholinergic agents were not found to have an effect (although only antiparkinsonian anticholinergic agents were assessed). Conversely, treatment with cognitive enhancers was associated with increased risk of becoming a frequent faller during the first step of the multivariable analysis, but was no longer significant in the second block. These findings would suggest that cognitive enhancer treatment may function as a surrogate variable for cognitive dysfunction.

In this study, we identified history of DBS prior to baseline visit, and also DBS implemented between visits as a significant risk factor of conversion to frequent faller status. One may assume that DBS is a surrogate for greater disease severity; however, 
Table 2 Final multivariate model for prediction of conversion to frequent faller status before follow-up visit

\begin{tabular}{|c|c|c|c|c|c|}
\hline Variables & Coefficient & SEM & $p$ Value & OR & $95 \% \mathrm{Cl}$ for $\mathrm{OR}$ \\
\hline Female sex & 2.23 & 0.94 & 0.017 & 9.33 & $1.49-58.56$ \\
\hline Age * sex & -0.04 & 0.01 & 0.011 & 0.97 & $0.94-0.99$ \\
\hline Certainty of diagnosis $\geq 90 \%$ & -0.48 & 0.20 & 0.016 & 0.62 & $0.42-0.92$ \\
\hline Motor fluctuations at baseline & 0.40 & 0.14 & 0.006 & 1.49 & $1.12-1.97$ \\
\hline L-dopa at baseline & 0.58 & 0.26 & 0.023 & 1.78 & $1.08-2.94$ \\
\hline Antidepressant at baseline & 0.35 & 0.14 & 0.012 & 1.41 & $1.08-1.85$ \\
\hline History of DBS at baseline & 1.08 & 0.19 & $<0.0001$ & 2.93 & $2.04-4.23$ \\
\hline PDQ-39 summary score & 0.02 & 0.00 & $<0.0001$ & 1.02 & $1.02-1.03$ \\
\hline H\&Y stage 2 (ref: stage 1) & 0.56 & 0.29 & 0.051 & 1.75 & $1.00-3.07$ \\
\hline H\&Y stage 3 (ref: stage 1 ) & 0.89 & 0.31 & 0.004 & 2.43 & $1.33-4.42$ \\
\hline Semantic fluency & -0.05 & 0.01 & $<0.0001$ & 0.95 & $0.93-0.98$ \\
\hline Added amantadine at baseline & 0.87 & 0.37 & 0.018 & 2.39 & $1.16-4.93$ \\
\hline Occupational therapy referral & 0.40 & 0.19 & 0.036 & 1.49 & $1.03-2.15$ \\
\hline Social work/counseling referral & 0.44 & 0.22 & 0.041 & 1.56 & $1.018-2.39$ \\
\hline DBS (between visits) & 0.62 & 0.31 & 0.045 & 1.85 & $1.01-3.38$ \\
\hline Cancer (between visits) & 0.28 & 0.12 & 0.022 & 1.32 & $1.04-1.67$ \\
\hline Osteoarthritis (between visits) & 0.20 & 0.07 & 0.006 & 1.22 & $1.06-1.41$ \\
\hline Increase in ED visits & 0.20 & 0.07 & 0.003 & 1.22 & $1.07-1.39$ \\
\hline Increase in trauma admissions & 0.50 & 0.20 & 0.013 & 1.65 & $1.11-2.46$ \\
\hline
\end{tabular}

Abbreviations: $\mathrm{Cl}$ = confidence interval; DBS = deep brain stimulation; $\mathrm{ED}=$ emergency department; H\&Y = Hoehn \& Yahr; OR = odds ratio; PDQ-39 = 39-Item Parkinson's Disease Questionnaire.

postural instability and falls may worsen within the year following the procedure ${ }^{31,32}$ and it seems that subthalamic nucleus DBS and globus pallidus pars interna DBS improve gait measures and quiet standing postural control in patients with $\mathrm{PD}$, but have no effect on or may even aggravate dynamic postural control. ${ }^{33}$ It is therefore becoming increasingly plausible that DBS may be an independent risk factor for falling in PD.
Among the measures of disease severity and quality of life, worse scores in PDQ-39 and semantic fluency and H\&Y stages 2 and 3 (as compared to H\&Y stage 1) conferred a higher risk of becoming a frequent faller. When we looked at the instruments alone, without consideration for any other variables, also MCSI, and, in addition to H\&Y stages 2 and 3, H\&Y stage 4/5 (as compared to H\&Y stage 1) now became significant, while

Table 3 Multivariate model of relative predictive values of questionnaires and assessments obtained at baseline

\begin{tabular}{|c|c|c|c|c|c|}
\hline Variable & Coefficient & SEM & $p$ Value & OR & $95 \% \mathrm{Cl}$ for $\mathrm{OR}$ \\
\hline PDQ-39 summary score & 0.027 & 0.00435 & $<0.0001$ & 1.027 & $1.019-1.036$ \\
\hline MCSI & 0.0147 & 0.00513 & 0.0042 & 1.015 & $1.005-1.025$ \\
\hline H\&Y stage 2 (ref:1) & 0.8583 & 0.2701 & 0.0015 & 2.359 & $1.389-4.006$ \\
\hline H\&Y stage 3 (ref:1) & 1.3633 & 0.2822 & $<0.0001$ & 3.909 & $2.249-6.796$ \\
\hline H\&Y stage 4/5 (ref:1) & 0.945 & 0.3693 & 0.0105 & 2.573 & $1.248-5.306$ \\
\hline Semantic fluency & -0.0521 & 0.0104 & $<0.0001$ & 0.949 & $0.93-0.969$ \\
\hline
\end{tabular}

Abbreviations: $\mathrm{Cl}$ = confidence interval; H\&Y = Hoehn \& Yahr; MSCl = Modified Caregiver Strain Index; OR = odds ratio; PDQ-39= 39-item Parkinson's Disease Questionnaire. 
TUG and immediate and 5-minute 5-word recall remained nonsignificant. The higher PDQ-39 and MCSI scores are surrogate measures of a greater overall disease burden. Regarding disease stage, maximum risk was associated with $\mathrm{H} \& \mathrm{Y}$ stage 3, likely because of reduced ambulatory activity (hence reduced exposure to falls) and increased use of gait assistive devices in stages 4 and 5. TUG, a measure of gross mobility and balance, failed to identify patients at higher risk to become frequent fallers. TUG is essentially a measure of speed of movement, and it is conceivable that reduced speed may improve stability and reduce the risk of falling. In addition, TUG is a measure of ambulatory ability, while a large proportion of falls in PD do not happen during ambulation. ${ }^{34,35}$

Semantic fluency was more strongly associated with increased risk for becoming a frequent faller than 5-word retention and recall. This cognitive measure is more closely associated with frontal function, ${ }^{36}$ and the association may reflect a greater role of frontal (executive) dysfunction in the genesis of falls as opposed to temporal (amnestic) dysfunction. In support of this hypothesis, non-PD older adults who fall demonstrate poorer performance in tests of frontal function than controls. ${ }^{37}$ Alternatively, this finding may reflect a greater association of falls with a cognitive subtype in $\mathrm{PD}$, without necessarily a causative relationship.

Besides referral for DBS, other treatment changes instituted during baseline visit, or health services utilized between visits that were significantly associated with a higher risk for becoming a frequent faller, included the addition of amantadine, referral to occupational therapy, a referral to social services, and increased use of emergency medical services and hospitalizations. We believe these variables to be surrogate markers of a specific phase in PD progression, namely the emergence of motor complications (particularly dyskinesia, the likely reason for initiating treatment with amantadine), failing global health (increase in utilization of medical services), increasing difficulties with activities of daily living (referral to occupational therapy), and increasing difficulty with independent function (a likely trigger for referral to social services).

Emergent osteoarthritis, a known risk factor for falling among older adults, ${ }^{38}$ and emergent cancer were associated with a higher risk for becoming a frequent faller. At least one possible common mechanism of falling among cancer patients would be chemotherapy-induced peripheral neuropathy. ${ }^{39}$ Regardless of specific underlying mechanisms, our findings highlight the need for seeking out, identifying, and addressing non-PDrelated causes of falls in persons with PD.

Our study has a number of limitations. The data are derived from a patient registry, and lack the granularity that would be necessary to identify more robust and precise associations. Specifically, the main outcome measure was obtained via history and lacked detail as to the nature and mechanism of falling. It is generally accepted that falls in PD are a heterogeneous phenomenon, and using a simple outcome measure may obscure mechanism-specific risk factors. In addition, the historical nature of the main outcome mitigates its reliability. We justify its use by the fact that this information is collected in a realistic manner, essentially mimicking clinical practice. The shortcomings of the primary outcome measure are largely responsible for the somewhat disappointing coefficient of determination of the final model (approximately $21 \%)$. Potentially important risk factors are unavailable through the NPF-QII database, including, and not limited to, exposure (i.e., the amount and type of locomotor activity in which individual patients engage), orthostatic hypotension, freezing of gait, and type and consistency of use of gait assistive devices. The NPF-QII database, although populous, may be subject to selection bias, which would influence the current analysis and the generalizability of the conclusions. Finally, this analysis provides associations between predictive variables and the main outcome; however, it does not prove causality, the results being subject to some degree of speculative interpretation.

The selection of "frequent falls" rather than "any falls" as the primary outcome merits additional discussion. The reference period for the NPF-QII registry question on falls is the 3 months preceding the visit, hence, a "no falls" response essentially translates to "no falls during the last 3 months," while "rare falls" would translate to "no more than 2 falls in the last 3 months," since 3 falls in the last 3 months would be categorized under "monthly falls." Considering that $35 \%$ of community-dwelling older adults report at least 1 fall a year, ${ }^{40}$ and given the specific classification adopted by the NPF-QII registry, we concluded that grouping "rare fallers" with "nonfallers" would optimize misclassification bias.

We have identified a number of associations between disease characteristics, treatments, and comorbidities and emergent falls in PD. The cause-effect relationships between the observed associations need to be further clarified; however, these characteristics may be used to identify individuals at high risk for preemptive fall prevention interventions, or for participation in randomized prospective controlled trials to evaluate proposed interventions.

\section{Author contributions}

S.A. Parashos: design of study, analysis and interpretation of the data, drafting and revising the manuscript. B.R. Bloem: analysis and interpretation of the data, revising the manuscript. N.M. Browner: analysis and interpretation of the data, revising the manuscript. N. Giladi: analysis and interpretation of the data, revising the manuscript. T. Gurevich: design of study, analysis and interpretation of the data, revising the manuscript. J.M. Hausdorff: analysis and interpretation of the data, revising the manuscript. Y. He: analysis and interpretation of the data, revising the manuscript. K.E. Lyons: analysis and interpretation of the data, revising the manuscript. Z. Mari: analysis and interpretation of the data, revising the manuscript. J.C. Morgan: analysis and interpretation of the data, revising the manuscript. B. Post: analysis and interpretation of the data, 
revising the manuscript. P. Schmidt: design of study, revising the manuscript. C.L. Wielinski: analysis and interpretation of the data, revising the manuscript. S.S. Wu: design of study, analysis and interpretation of the data, revising the manuscript. S.S. Wu and Y. He were responsible for the statistical design and performed the statistical analyses.

\section{Study funding}

Supported by the National Parkinson Foundation.

\section{Disclosure}

S.A. Parashos has received funding for travel from National Parkinson Foundation and NIH; receives publishing royalties from Navigating Life With Parkinson Disease (Oxford University Press, 2013); serves as a consultant for Dong A; receives research support from Astellas, Sunovion, Acorda, Impax, Pharma2B, Adamas, Biotie, Cynapsus, PCORI, NIH/NINDS, Park Nicollet Foundation, National Parkinson Foundation, Struthers Parkinson's Center, and Michael J. Fox Foundation; and holds stock/ stock options in St. Jude Medical and Abbott Laboratories. B. R. Bloem serves on scientific advisory boards for Michael J. Fox Foundation, Parkinson Vereniging, Movement Disorder Society, and Brian Grant Foundation; has received speaker honoraria from AbbVie, Zambon, Bial, and Kyowa Kirin; serves as an Associate Editor of Journal of Parkinson's Disease; serves as a consultant for Zambon; and receives research support from UCB, AbbVie, Verily, Netherlands Organization for Scientific Research, Michael J. Fox Foundation, Parkinson Vereniging, Prinses Beatrix Foundation, Stichting Parkinson Fonds, National Parkinson Foundation, and Hersenstichting. N.M. Browner has received funding for travel from the Parkinson Foundation and receives research support from National Center for Research Resources, NIH/The North Carolina Translational and Clinical Sciences Institute, Parkinson Foundation Center of Excellence, and Parkinson Foundation Community Grants. N. Giladi serves on scientific advisory boards for Teva-Lundbeck, LTI, Dexel, AbbVie, NeuroDerm, and Sionara Mont4; has received funding for travel and speaker honoraria from Teva, Novartis, UCB, AbbVie, Shaie, Genzyme, Bial, Movement Disorder Society, and Asian Neuro-rehabilitation Society; serves as Associate Editor for the Journal of Neural Transmission and Current Treatment Opinion in Neurology and on the Editorial Board of Journal of Parkinson's disease; has served as a consultant for Teva, IntecPharma, NeuroDerm, Biogen, Pharma2B, Denali, Abbvie, Monfort, and UCB; serves on the speakers' bureau for TevaLundbeck and UCB; and receives research support from Israel Science Foundation, NIH, Michael J. Fox Foundation, and National Parkinson Foundation. T. Gurevich has received funding for travel and/or speaker honoraria from AbbVie, Medtronic, Allergan, Medisson, and Parkinson's Foundation; serves as a consultant for Abbvie and Neuroderm; and receives research support from Parkinson's Foundation and Israel Innovation Authority. J.M. Hausdorff has received funding for travel from the Movement Disorders Society and the University of Michigan; serves on the editorial boards of Movement Disorders and Frontiers in Neurology: Movement Disorders and as an Associate Editor for Journal of Gerontology Medical Sciences and
Journal of NeuroEngineering and Rehabilitation; is author on a patent re: application on the use of virtual reality (submitted); the intellectual property rights are held by the Tel Aviv Medical Center; receives research support from European Commission, NIH, Michael J. Fox Foundation, NMMS, and BSF; and holds stock/stock options in Teva. Y. He reports no disclosures. K.E. Lyons serves on scientific advisory boards for Sage Therapeutics, St Jude Medical, and Acorda Therapeutics; is past CoEditor-in-Chief of the International Journal of Neuroscience; serves on the speakers' bureau for ACADIA Pharmaceuticals; and serves on the National Parkinson Foundation Parkinson Outreach Project steering committee. Z. Mari serves as an Associate Editor for Parkinsonism and Related Disorders; serves as a consultant for GB Sciences; receives research support from AbbVie, Great Lakes Neurotechnology, Avid Radiopharmaceuticals, NIH (NHLBI, NINDS, National Parkinson Foundation, Johns Hopkins Parkinson's Disease Center of Excellence, and Michael J. Fox Foundation; and has participated in medico-legal cases. J.C. Morgan has received speaker honoraria from Acadia, Impax, Neurocrine, National Parkinson Foundation, and Teva; serves as a consultant for AbbVie, Acadia, Adamas, Neurocrine, Parkinson's Foundation, and Teva; serves on speakers' bureaus for Acadia, Adamas, Impax, Parkinson's Foundation, Neurocrine, and Teva; receives research support from AbbVie, Acorda, American College of Radiology, CHDI, Kyowa, Lilly, Lundbeck, Serina, US World Meds, VA Merit Review, National Parkinson Foundation, Parkinson's Outcome Project Grant, and Parkinson's Study Group; and has participated in medico-legal cases. B. Post reports no disclosures. P.N. Schmidt receives research support from National Parkinson Foundation and Parkinson's Foundation. C.L. Wielinski reports no disclosures. S.S. Wu is author on a pending patent re: a computer- implemented method for preserving privacy for data collection, publication, and analysis; serves as a consultant for Bioness Inc.; and receives research support from NIH (NIMH, NINDS, NIA, NIAMS, NIGMS), US Department of Veterans Affairs, and US Army Department of Defense. Full disclosure form information provided by the authors is available with the full text of this article at Neurology.org/cp.

Received December 13, 2017. Accepted in final form March 27, 2018.

\section{References}

1. Wielinski CL, Erickson-Davis C, Wichmann R, Walde-Douglas M, Parashos SA. Falls and injuries resulting from falls among patients with Parkinson's disease and other parkinsonian syndromes. Mov Disord 2005;20:410-415.

2. Schrag A, Jahanshahi M, Quinn NP. What contributes to depression in Parkinson's disease? Psychol Med 2001;31:65-73.

3. Melton LJ III, Leibson CL, Achenbach SJ, et al. Fracture risk after the diagnosis of Parkinson's disease: influence of concomitant dementia. Mov Disord 2006;21: 1361-1367.

4. Allyson Jones C, Wayne Martin WR, Wieler M, King-Jesso P, Voaklander DC. Incidence and mortality of Parkinson's disease in older Canadians. Parkinsonism Relat Disord 2010;18:327-331.

5. Schrag A, Hovris A, Morley D, Quinn N, Jahanshahi M. Caregiver-burden in Parkinson's disease is closely associated with psychiatric symptoms, falls, and disability. Parkinsonism Relat Disord 2006;12:35-41.

6. Kaltenboeck A, Johnson SJ, Davis MR, et al. Direct costs and survival of Medicare beneficiaries with early and advanced Parkinson's disease. Parkinsonism Relat Disord 2012;18:321-326.

7. Rahman S, Griffin HJ, Quinn NP, Jahanshahi M. Quality of life in Parkinson's disease: the relative importance of the symptoms. Mov Disord 2008;23:1428-1434.

8. Pickering RM, Grimbergen YA, Rigney U, et al. A meta-analysis of six prospective studies of falling in Parkinson's disease. Mov Disord 2007;22:1892-1900. 
9. Wood BH, Bilclough JA, Bowron A, Walker RW. Incidence and prediction of falls in Parkinson's disease: a prospective multidisciplinary study. J Neurol Neurosurg Psychiatry 2002;72:721-725.

10. Balash Y, Peretz C, Leibovich G, Herman T, Hausdorff JM, Giladi N. Falls in outpatients with Parkinson's disease: frequency, impact and identifying factors. J Neurol 2005;252:1310-1315.

11. Parashos SA, Wielinski CL, Giladi N, Gurevich T. Falls in Parkinson disease: analysis of a large cross-sectional cohort. J Parkinsons Dis 2013;3:515-522.

12. Kataoka $\mathrm{H}$, Ueno $\mathrm{S}$. Low $\mathrm{FAB}$ score as a predictor of future falling in patients with Parkinson's disease: a 2.5-year prospective study. J Neurol 2015;262:2049-2055.

13. Weiss A, Herman T, Giladi N, Hausdorff JM. Objective assessment of fall risk in Parkinson's disease using a body-fixed sensor worn for 3 days. PLoS One 2014;9:e96675.

14. Mak MK, Auyeung MM. The mini-BESTest can predict parkinsonian recurrent fallers: a 6-month prospective study. J Rehabil Med 2013;45:565-571.

15. Heinzel S, Maechtel M, Hasmann SE, et al. Motor dual-tasking deficits predict falls in Parkinson's disease: a prospective study. Parkinsonism Relat Disord 2016;26:73-77.

16. Okun MS, Siderowf A, Nutt JG, et al. Piloting the NPF data-driven quality improvement initiative. Parkinsonism Relat Disord 2010;16:517-521.

17. Peto V, Jenkinson C, Fitzpatrick R, Greenhall R. The development and validation of a short measure of functioning and well being for individuals with Parkinson's disease. Qual Life Res 1995;4:241-248.

18. Hoehn MM, Yahr MD. Parkinsonism: onset, progression and mortality. Neurology 1967; 17:427-442.

19. Morris S, Morris ME, Iansek R. Reliability of measurements obtained with the Timed "Up \& Go" test in people with Parkinson disease. Phys Ther 2001;81:810-818.

20. Stull DE. The Multidimensional Caregiver Strain Index (MCSI): its measurement and structure. J Clin Geropsychol 1996;2:175-196.

21. Nasreddine ZS, Phillips NA, Bédirian V, et al. The Montreal Cognitive Assessment, MoCA: a brief screening tool for mild cognitive impairment. J Am Geriatr Soc 2005; 53:695-699.

22. Chou KL, Elm JJ, Wielinski CL, et al. Factors associated with falling in early, treated Parkinson's disease: the NET-PD LE-1 cohort. J Neurol Sci 2017;377:137-143.

23. Gazibara T, Tepavcevic DK, Svetel M, et al. Recurrent falls in Parkinson's disease after one year of follow-up: a nested case-control study. Arch Gerontol Geriatr 2016;65:17-24.

24. van der Heeden JF, Marinus J, Martinez-Martin P, Rodriguez-Blazquez C, Geraedts VJ, van Hilten JJ. Postural instability and gait are associated with severity and prognosis of Parkinson disease. Neurology 2016;86:2243-2250.

25. Marcum ZA, Perera S, Thorpe JM, et al. Antidepressant use and recurrent falls in community-dwelling older adults: findings from the Health ABC study. Ann Pharmacother 2016;50:525-533.
26. Masud T, Frost M, Ryg J, et al. Central nervous system medications and falls risk in men aged 60-75 years: the Study on Male Osteoporosis and Aging (SOMA). Age Ageing 2013;42:121-124

27. Menant JC, Wong AK, Trollor JN, Close JC, Lord SR. Depressive symptoms and orthostatic hypotension are risk factors for unexplained falls in community-living older people. J Am Geriatr Soc 2016;64:1073-1078.

28. Burn DJ, Landau S, Hindle JV, et al. Parkinson's disease motor subtypes and mood. Mov Disord 2012;27:379-386.

29. Crispo JAG, Willis AW, Thibault DP, et al. Associations between anticholinergic burden and adverse health outcomes in Parkinson disease. PLoS One 2016;11: e0150621.

30. Chung KA, Lobb BM, Nutt JG, Horak FB. Effects of a central cholinesterase inhibitor on reducing falls in Parkinson disease. Neurology 2010;75:1263-1269.

31. Guehl D, Cuny E, Benazzouz A, et al. Side-effects of subthalamic stimulation in Parkinson's disease: clinical evolution and predictive factors. Eur J Neurol 2006;13:963-971.

32. Weaver FM, Follett K, Stern M, et al. Bilateral deep brain stimulation vs best medical therapy for patients with advanced Parkinson disease: a randomized controlled trial. JAMA 2009;301:63-73.

33. Collomb-Clerc A, Welter ML. Effects of deep brain stimulation on balance and gait in patients with Parkinson's disease: a systematic neurophysiological review. Neurophysiol Clin 2015;45:371-388.

34. Parashos SA, Erickson-Davis C, Krohn SA, Wielinski CL. Gait and balance initiative (GABI): phenomenology of falls in Parkinson's disease. Mov Disord 2008;23(suppl 1): S332-S333.

35. Ashburn A, Stack E, Ballinger C, Fazakarley L, Fitton C. The circumstances of falls among people with Parkinson's disease and the use of falls diaries to facilitate reporting. Disabil Rehabil 2008;30:1205-1212.

36. Flowers KA, Robertson C, Sheridan MR. Some characteristics of word fluency in Parkinson's disease. J Neurolinguist 1995;9:33-46.

37. Hausdorff JM, Doniger GM, Springer S, Yogev G, Simon ES, Giladi N. A common cognitive profile in elderly fallers and in patients with Parkinson's disease: the prominence of impaired executive function and attention. Exp Aging Res 2006;32: 411-429.

38. Campbell AJ, Borrie MJ, Spears GF. Risk factors for falls in a community-based prospective study of people 70 years and older. J Gerontol 1989;44:M112-M117.

39. Kolb NA, Smith AG, Singleton JR, et al. The association of chemotherapy-induced peripheral neuropathy symptoms and the risk of falling. JAMA Neurol 2016;73: 860-866.

40. Blake AJ, Morgan K, Bendall MJ, et al. Falls by elderly people at home: prevalence and associated factors. Age Ageing 1988;17:365-372.

\section{Practical Implications}

Neurology ${ }^{\oplus}$ Clinical Practice is committed to providing clinical insights helpful to neurologists in everyday practice. Each Full Case includes a "Practical Implications" statement, a pearl of wisdom for the practicing clinician. 